\title{
Pavement rehabilitation and maintenance prioritization of urban roads using fuzzy logic.
}

\begin{abstract}
With regard to limits in budget, inevitably, one must choose prioritized projects in pavements rehabilitation and maintenance process. This paper looks into prioritization based upon a model including all effects of important factors like pavement condition index, traffic volume, road width as well as rehabilitation and maintenance cost. Since defining a model that introduces all those factors was difficult, a more advanced modeling named fuzzy logic was referred for the problem of prioritization. Although analytical hierarchy process can be used for decision making process as well, fuzzy modeling lets one have more precise choices for the outcome. Finally with the help of MATLAB software and coded M-files, inference engines such as Product engine, Dienes-Rescher and Lukasiewicz were all tested and the logical favorite separation for this application was found in product inference engine. As a case study some streets located in district No. 6 of Tehran municipality were selected and the favorite mathematical model was executed on those streets. This model was used for prioritizing these 131 sections.
\end{abstract}

Keyword: Fuzzy logic; Inference engines; Pavement maintenance; Maintenance cost; Traffic volume; Roadway width; Pavement condition index. 